\title{
Temporal trends in rates of infection-related hospitalisations in Hong Kong people with and without diabetes, 2001-2016: a retrospective study
}

\author{
Andrea O. Y. Luk ${ }^{1,2}$ (D) Hongjiang Wu ${ }^{1}$ (D) Eric S. H. Lau ${ }^{1} \cdot$ Aimin Yang ${ }^{1} \cdot$ Wing-Yee So ${ }^{3} \cdot$ Elaine Chow ${ }^{1}$. \\ Alice P. S. Kong ${ }^{1,2} \cdot$ David S. C. Hui $^{1}$ (1) $\cdot$ Ronald C. W. Ma ${ }^{1,2}$ (1) $\cdot$ Juliana C. N. Chan ${ }^{1,2}$ (D)
}

Received: 27 May 2020 / Accepted: 10 August 2020 / Published online: 28 September 2020

(C) Springer-Verlag GmbH Germany, part of Springer Nature 2020

\begin{abstract}
Aims/hypothesis Infection is an under-recognised but important complication in people with diabetes. Studies on temporal trends in incidence of infection in this population are limited. We report the trends in infection-related hospitalisation in people with diabetes and compared hospitalisation rates between people with and without diabetes in Hong Kong.

Methods Hospital admissions with infection, including pneumonia, influenza, tuberculosis, kidney infection, urinary tract infection, cellulitis, osteomyelitis, foot infection and sepsis, listed as principal diagnosis occurring between 2001 and 2016 were identified from people with diabetes in the electronic medical record system of the Hong Kong Hospital Authority. Data on hospitalisation for a subset of these infections in the general population between 2007 and 2016 were obtained from the Department of Health. The number of people with diabetes ranged between 117,322 in 2001 and 570,929 in 2016, and the number without diabetes ranged between $5,242,614$ in 2007 and 5,593,153 in 2016. Joinpoint regression was used to describe the trends.

Results In people with diabetes, over a period of 16 years, the age-standardised annual rates of hospitalisation decreased for tuberculosis but increased for influenza; rates of hospitalisation for pneumonia increased up until 2004/2005 and declined in men and stabilised in women. The rates of hospitalisation for most infection types were unchanged or increased in the 20-44 year and 45-64 year age groups and decreased in those aged 65 years or above. Trends for most of the infections were similar when comparing sexes. Between 2007 and 2016, the rate ratios of hospitalisation for most infection types between people with and without diabetes were stable, and the rate ratios remained higher in people with diabetes, ranging from 1.3-1.4 for pneumonia to 3.2-4.9 for kidney infections in 2016 compared with non-diabetic individuals.

Conclusions/interpretation Despite advances in medical care, hospitalisation due to infections remains a major healthcare burden in people with diabetes.
\end{abstract}

Keywords Cellulitis $\cdot$ Diabetes $\cdot$ Hospitalisation $\cdot$ Infection $\cdot$ Influenza $\cdot$ Kidney and urinary tract infection $\cdot$ Osteomyelitis · Pneumonia $\cdot$ Sepsis $\cdot$ Tuberculosis

Electronic supplementary material The online version of this article (https://doi.org/10.1007/s00125-020-05286-2) contains peer-reviewed but unedited supplementary material, which is available to authorised users.

Andrea O. Y. Luk

andrealuk@cuhk.edu.hk

1 Department of Medicine and Therapeutics, The Chinese University of Hong Kong, Hong Kong, Hong Kong Special Administrative Region, People's Republic of China

2 Li Ka Shing Institute of Health Sciences, The Chinese University of Hong Kong, Hong Kong, Hong Kong Special Administrative Region, People's Republic of China

3 Hong Kong Hospital Authority, Hong Kong, Hong Kong Special Administrative Region, People's Republic of China

\author{
Abbreviations \\ AAPC Average annual percentage change \\ APC Annual percentage change \\ COVID-19 Coronavirus disease 2019 \\ HKHA Honk Kong Hospital Authority \\ HKDSD Hong Kong Diabetes Surveillance Database \\ ICD International Classification of Diseases \\ SARS Severe acute respiratory syndrome
}

\section{Introduction}

People with diabetes have increased susceptibility to most types of infectious diseases. Previous studies reported higher 


\section{Research in context}

\section{What is already known about this subject?}

- Infection is an important complication in diabetes but few studies have examined the temporal trends of infection or infection-related hospitalisation in this population

- One recent study conducted in the USA indicated stable or increasing incidence rates of most infection types in people with diabetes but similar surveillance studies have not been undertaken elsewhere

\section{What is the key question?}

- What is the trend in infection-related hospitalisation in people with diabetes and how does this compare with infection rates in people without diabetes?

\section{What are the new findings?}

- In people with diabetes in Hong Kong, the rates of hospitalisation for influenza increased, rates for tuberculosis and urinary tract infection decreased, and rates for other infection types were stable or fluctuated

- We detected an age differential in the trends for hospitalisation for infection. In the young to middle-aged groups, hospitalisation rates for most infection types increased or remained unchanged, while in older age groups, infection rates tended to decline over time

- Across the surveillance period, rates of hospitalisation for all infection types remained consistently higher in people with vs without diabetes. The relative difference narrowed for rates of pneumonia but were unchanged for other infection types

\section{How might this impact on clinical practice in the foreseeable future?}

- The lack of decline in the rates of diabetes-related infection indicate that current practice targeting control of blood glucose and other diabetes indices have not lowered the risk of serious infection. Additional measures directed towards awareness, prevention and treatment of infection are urgently required in this population

risks for the following infections in people with vs without diabetes: 1.2- to 2.6-fold for pneumonia [1, 2]; 2.0- to 3.2-fold for sepsis [2-4]; 3.0- to 4.3-fold for urinary tract infection [2, 4]; and 1.8- to 2.0 -fold for cellulitis [3, 5]. People with diabetes are also more likely to have severe clinical presentation and to succumb after acquiring an infection. For instance, mortality rates due to sepsis was twice as high among people with diabetes than their counterparts without diabetes [6]. In a retrospective analysis of individuals who had acquired the severe acute respiratory syndrome (SARS) coronavirus in China, coexisting diabetes increased the age- and sex-adjusted odds of death by threefold, with a strong correlation between blood glucose and disease severity [7]. The high death rates in people with diabetes during the recent coronavirus disease 2019 (COVID-19) pandemic, especially in those with severe inhospital hyperglycaemia, highlight the vulnerability of diabetic individuals during acute emergencies [8]. The underlying causes for the increased rates of infection in diabetes are multifactorial. Hyperglycaemia has inhibitory effects on many components of the immune system, hampers pathogen clearance and affects host inflammatory response $[9,10]$. Moreover, diabetes is closely related to other medical conditions such as peripheral artery disease, sensory neuropathy and others that lower barrier defence against infectious agents [11,12].
Contemporary studies on the temporal trends of diabetesrelated complications found large declines in the rates of these clinical events in people with diabetes, driven primarily by improvements in risk factor control [13-15]. However, a recent study in the USA found that while rates of infection-related hospitalisation remained unchanged in people with diabetes, they declined in people without diabetes [2]. This suggests that measures to improve care in people with diabetes may not have been as effective in preventing infection and/or worsening of its clinical course. To our knowledge, the study in the USA is the only large-scale epidemiological study evaluating trends for infection in people with diabetes and it is not known whether similar trends are apparent in other populations. Here, we describe temporal changes in rates of infection-related hospitalisation using a territory-wide database of people with diabetes in Hong Kong spanning a 16 year period from 2001 to 2016. We further compared infection rates between people with diabetes and those without diabetes in Hong Kong from 2007 to 2016.

\section{Methods}

Study population The Hong Kong Hospital Authority (HKHA) is a statutory body established in 1990 and governs 
all the public hospitals as well as specialist and general outpatient clinics to provide about $95 \%$ of inpatient services for Hong Kong residents [16]. The Hong Kong Diabetes Surveillance Database (HKDSD) is a population-based cohort of people with diabetes identified from the HKHA electronic medical record system between 1 January 2000 and 31 December 2016. Details of the HKDSD have been described previously [17]. In brief, individuals were included in the database if they fulfil laboratory criteria for diabetes $\left(\mathrm{HbA}_{1 \mathrm{c}}\right.$ $\geq 48 \mathrm{mmol} / \mathrm{mol}[6.5 \%]$ or fasting plasma glucose $\geq 7.0 \mathrm{mmol} /$ 1 ), were ever prescribed insulin or non-insulin glucose-lowering drugs, and/or were coded to have diabetes by their physicians. The database excluded women with gestational diabetes.

To compare the rates of infection-related hospitalisation between people with and without diabetes, summary statistics of hospitalisation for infective causes among the general Hong Kong population were obtained from the Department of Health. Data at the Department of Health were provided by the HKHA. Data on the number of people hospitalised stratified by sex and 5 year age interval in each calendar year over a 10 year period between 1 January 2007 and 31 December 2016 were available; data before 2007 were not available. The analysis was restricted to people aged 20 years or above. This study was approved by the local clinical research ethics committee.

Events ascertainment Infection-related hospitalisations for people in the HKDSD were identified using International Classification of Diseases (ICD), Ninth Revision (ICD-9; http://www.icd9data.com/2007/Volume1/default.htm) codes listed as the principal diagnosis in the hospital discharge summary. Infection types reported in previous literature to be more prevalent in people with diabetes were considered, including pneumonia, influenza, tuberculosis, kidney infection, urinary tract infection, cellulitis, osteomyelitis, foot infection and sepsis (electronic supplementary material [ESM] Table 1) [1-5]. Infection-related hospitalisation for the Hong Kong general population were identified based on ICD-10 codes (http://apps.who.int/classifications/icd10/ browse/2016/en) recorded as the principal diagnosis. Data on pneumonia, influenza, tuberculosis, kidney infection and sepsis were available (ESM Table 1).

Statistical analysis We calculated age-standardised annual rates of infection-related hospitalisations for people with diabetes between 2001 and 2016, and for people without diabetes between 2007 and 2016. We counted the number of infection-related hospitalisations as the numerator and used the mid-year population for people with/without diabetes as the denominator in each study year. All rates were agestandardised to the 2016 Hong Kong Census mid-year population. We subtracted the number of infection-related hospitalisations in the HKDSD from the total number of infection-related hospitalisations in the general Hong Kong population to obtain the number of infection-related hospitalisations in people without diabetes in Hong Kong. A similar method was used to obtain the mid-year population estimates for people without diabetes by subtracting the number for the mid-year population in the HKDSD from the mid-year population estimates for the whole Hong Kong population reported by the Hong Kong Census and Statistics Department. We used joinpoint regression models to describe the trends in rates of infection-related hospitalisations. The joinpoint regression model uses a Monte Carlo permutation test to detect whether there is a significant change in trends during the study period. The annual percentage change (APC) was reported for each linear trend segment detected from the joinpoint regression model and the average annual percentage change (AAPC) was reported for the entire study period. We used Poisson regression models to calculate the rate ratios of infection-related hospitalisations comparing people with and without diabetes, with adjustment for age and stratification by sex and study year. We undertook a sensitivity analysis excluding people who were newly included in the HKDSD in each study year to assess the effect of incident diabetes on infection trends. All analyses were performed using R (version 3.5.3; Vienna, Austria) or Joinpoint Regression Program (version 4.7.0.0; Statistical Methodology and Applications Branch, Surveillance Research Program, National Cancer Institute, Bethesda, MD, USA). A two-tailed $p$ value less than 0.05 was considered statistically significant.

\section{Results}

Infection-related hospitalisation in people with diabetes The HKDSD included 390,071 men and 380,007 women aged 20 years or above with diabetes. Between 2001 and 2016, 159,438 hospitalisations for pneumonia, 10,872 for influenza, 16,463 for tuberculosis, 3763 for kidney infection, 93,252 for urinary tract infection, 45,470 for cellulitis, 2625 for osteomyelitis, 30,428 for foot infections and 41,942 for sepsis were recorded (ESM Tables 2, 3). During the study period, men had higher rates of hospitalisation for pneumonia, tuberculosis, cellulitis, osteomyelitis and foot infection, and women had higher rates of hospitalisation for kidney infection and urinary tract infection (Table 1). The rates for influenza and sepsis were similar between the sexes (Table 1). In both men and women, rates of most infection types increased with increasing age, except for cellulitis, which occurred most frequently in the youngest age group of 20-44 years in men (ESM Tables 4, Table 5).

Trends in rates of infection-related hospitalisation by sex in people with diabetes For pneumonia, an increase in 
Table 1 Joinpoint analysis of trends in age-standardised rates of hospitalisation for infection in men and women with diabetes

\begin{tabular}{|c|c|c|c|c|c|c|c|c|c|}
\hline \multirow[t]{2}{*}{ Complication } & \multicolumn{3}{|c|}{ Event rate (per 10,000 persons) $)^{a}$} & \multicolumn{2}{|c|}{ Time period 1} & \multicolumn{2}{|c|}{ Time period 2} & \multicolumn{2}{|c|}{$\begin{array}{l}\text { Event rate in joinpoint } \\
\text { year (per } 10,000 \text { persons) }\end{array}$} \\
\hline & 2001 & 2016 & AAPC $(95 \% \mathrm{CI})$ & Year & $\mathrm{APC}(95 \% \mathrm{CI})$ & Year & $\mathrm{APC}(95 \% \mathrm{CI})$ & Year & Rate \\
\hline \multicolumn{10}{|l|}{ Pneumonia } \\
\hline Men & 116.3 & 171.1 & $3.4(1.1,5.8)^{*}$ & 2001-2004 & $26.8(12.4,43.0)^{*}$ & $2004-2016$ & $-1.7(-2.6,-0.8)^{*}$ & 2004 & 186.3 \\
\hline Women & 72.4 & 102.4 & $4.1(0.7,7.6)^{*}$ & $2001-2005$ & $16.7(3.7,31.3)^{*}$ & $2005-2016$ & $-0.2(-2.8,2.6)$ & 2005 & 116.2 \\
\hline \multicolumn{10}{|l|}{ Influenza } \\
\hline Men & 0.4 & 18.6 & $14.8(8.0,22.1)^{*}$ & 2001-2016 & $14.8(8.0,22.1)^{*}$ & - & - & - & - \\
\hline Women & 0.6 & 19.7 & $16.6(8.7,25.1)^{*}$ & 2001-2016 & $16.6(8.7,25.1)^{*}$ & - & - & - & - \\
\hline \multicolumn{10}{|l|}{ Tuberculosis } \\
\hline Men & 133.2 & 25.7 & $-9.9(-14.3,-5.3)^{*}$ & $2001-2007$ & $-17.1(-24.9,-8.4)^{*}$ & $2007-2016$ & $-4.8(-10.8,1.7)$ & 2007 & 37.2 \\
\hline Women & 41.9 & 21.8 & $-9.3(-13.1,-5.4)^{*}$ & 2001-2016 & $-9.3(-13.1,-5.4)^{*}$ & - & - & - & - \\
\hline \multicolumn{10}{|c|}{ Kidney infection } \\
\hline Men & 3.0 & 5.1 & $4.6(-2.8,12.6)$ & 2001-2016 & $4.6(-2.8,12.6)$ & - & - & - & - \\
\hline Women & 15.8 & 27.1 & $6.0(-1.8,14.5)$ & 2001-2011 & $-1.8(-7.9,4.9)$ & 2011-2016 & $23.5(-1.3,54.5)$ & 2011 & 9.1 \\
\hline \multicolumn{10}{|c|}{ Urinary tract infection } \\
\hline Men & 98.4 & 53.2 & $-4.2(-5.9,-2.4)^{*}$ & 2001-2011 & $-5.9(-7.4,-4.4)^{*}$ & 2011-2016 & $-0.6(-5.7,4.8)$ & 2011 & 50.6 \\
\hline Women & 195.7 & 98.8 & $-3.8(-5.3,-2.3)^{*}$ & 2001-2016 & $-3.8(-5.3,-2.3)^{*}$ & - & - & - & - \\
\hline \multicolumn{10}{|l|}{ Cellulitis } \\
\hline Men & 210.9 & 134.9 & $-1.5(-3.5,0.5)$ & 2001-2016 & $-1.5(-3.5,0.5)$ & - & - & - & - \\
\hline Women & 94.4 & 91.6 & $1.0(-1.9,3.8)$ & 2001-2016 & $1.0(-1.9,3.8)$ & - & - & - & - \\
\hline \multicolumn{10}{|l|}{ Osteomyelitis } \\
\hline Men & 8.7 & 12.3 & $2.2(-2.9,7.5)$ & 2001-2016 & $2.2(-2.9,7.5)$ & - & - & - & - \\
\hline Women & 3.8 & 2.1 & $-3.3(-8.8,2.5)$ & 2001-2007 & $-13.1(-25.2,0.9)$ & $2007-2016$ & $3.8(-0.8,8.5)$ & 2007 & 1.3 \\
\hline \multicolumn{10}{|l|}{ Foot infection } \\
\hline Men & 72.6 & 44.4 & $-1.5(-3.1,0.2)$ & 2001-2016 & $-1.5(-3.1,0.2)$ & - & - & - & - \\
\hline Women & 44.9 & 19.5 & $-5.2(-6.7,-3.6)^{*}$ & 2001-2016 & $-5.2(-6.7,-3.6)^{*}$ & - & - & - & - \\
\hline \multicolumn{10}{|l|}{ Sepsis } \\
\hline Men & 56.1 & 39.6 & $-2.8(-5.1,-0.4)^{*}$ & 2001-2007 & $-10.5(-15.8,-4.9)^{*}$ & $2007-2016$ & $2.7(0.7,4.7)^{*}$ & 2007 & 29.9 \\
\hline Women & 48.1 & 34.7 & $-1.8(-5.2,1.7)$ & 2001-2006 & $-12.6(-20.1,-4.3)^{*}$ & 2006-2016 & $4.0(0.2,8.0)^{*}$ & 2006 & 25.2 \\
\hline
\end{tabular}

${ }^{a}$ The denominator for event rates was the mid-year population of people with diabetes in each study year (2001 and 2016)

$* p<0.05$; the tests of significance use a Monte Carlo permutation method

hospitalisation rate was detected between 2001 and 2004 in men (APC 26.8 [95\% CI 12.4, 43.0]) and between 2001 and 2005 in women (APC 16.7 [95\% CI 3.7, 31.3]), after which the rates declined in men (APC -1.7 [95\% CI $-2.6,-0.8])$ and were unchanged in women (Table 1, Fig. 1a). The hospitalisation rate for influenza showed a linear increasing trend in both sexes (Table 1, Fig. 1b). For tuberculosis, the decrease in rates was linear in women but in men the decrease was mainly observed between 2001 and 2007 (APC -17.1 $[95 \%$ CI $-24.9,-8.4]$ ) and stabilised thereafter (Table 1, Fig. 1i). Hospitalisation for sepsis showed a decreasing trend between 2001 and 2007 in men (APC -10.5 [95\% CI -15.8, $-4.9]$ ) and between 2001 and 2006 in women (APC -12.6 [95\% CI $-20.1,-4.3])$, followed by increases in rates over the remaining time period in both men (APC 2.7 [95\% 0.7, 4.7]) and women (APC 4.0 [95\% CI 0.2, 8.0]) (Table 1, Fig. 1h). The rate of hospitalisation for urinary tract infection declined between 2001 and 2011 (APC -5.9 [95\% -7.4, -4.4 ) and flattened out from 2011 to 2016 (APC -0.6 [95\% $\mathrm{CI}-5.7,4.8]$ ) in men; a linear declining trend was found in women over the whole period from 2001 to 2016 (APC -3.8 [95\% CI $-5.3,-2.3]$ ) (Table 1, Fig. 1d). No consistent time trend was observed for rates of hospitalisation for kidney infection, cellulitis, osteomyelitis or foot infection over the entire surveillance period (Table 1, Fig. 1c, e-g). Exclusion of people with newly diagnosed diabetes in each study year did not affect the trend estimates (ESM Fig. 1).

Trends in rates of infection-related hospitalisation by age groups in people with diabetes Age-stratified trend analysis was conducted for rates of hospitalisation for pneumonia, tuberculosis, urinary tract infection, cellulitis, foot infection 
Fig. 1 Age-standardised rates of hospitalisation (per 10,000 people) for pneumonia (a), influenza (b), kidney infection (c), urinary tract infection (d), cellulitis (e), foot infection (f), osteomyelitis (g), sepsis (h) and tuberculosis (i) in men (blue) and women (red) with diabetes in Hong Kong between 2001 and 2016
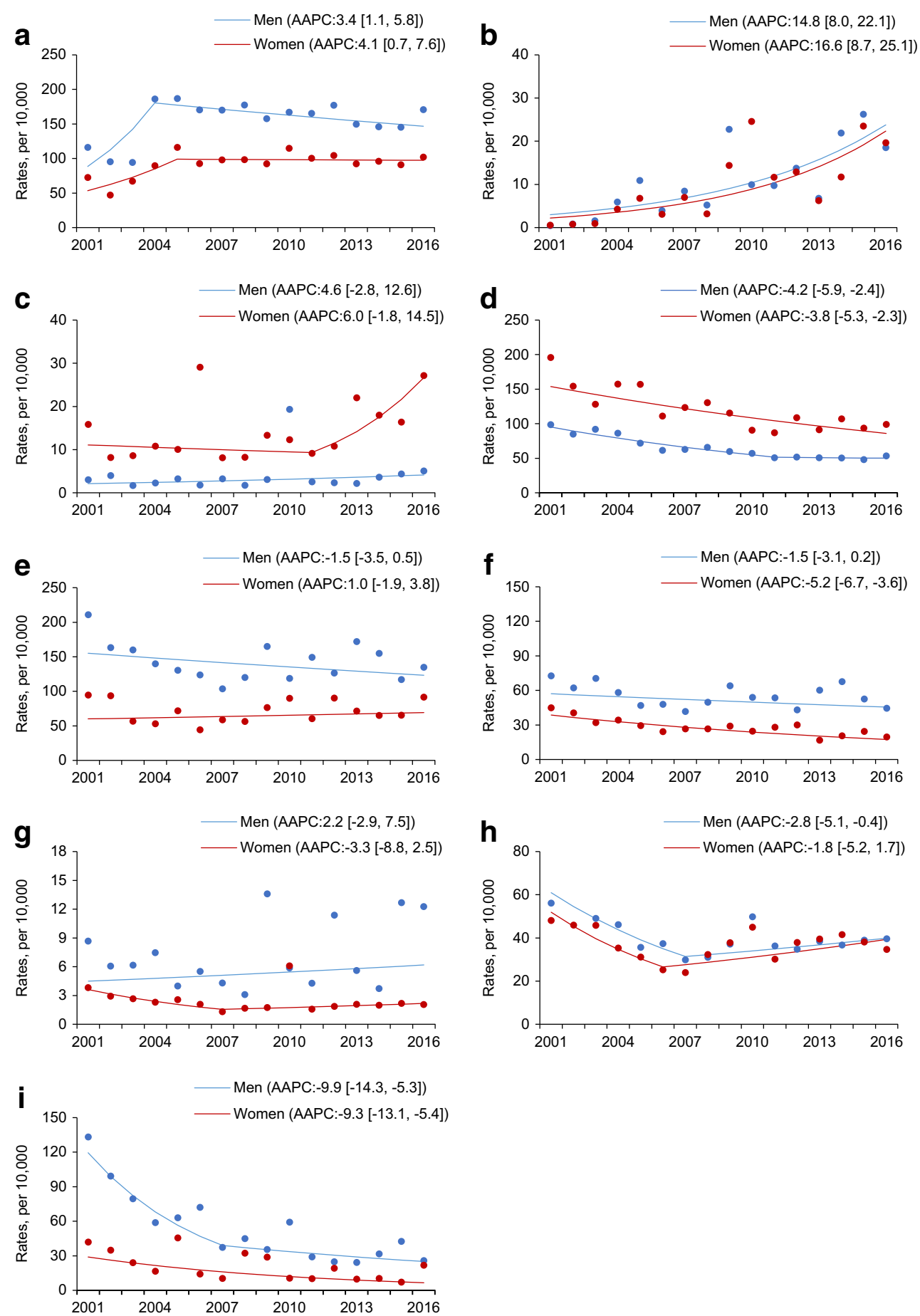
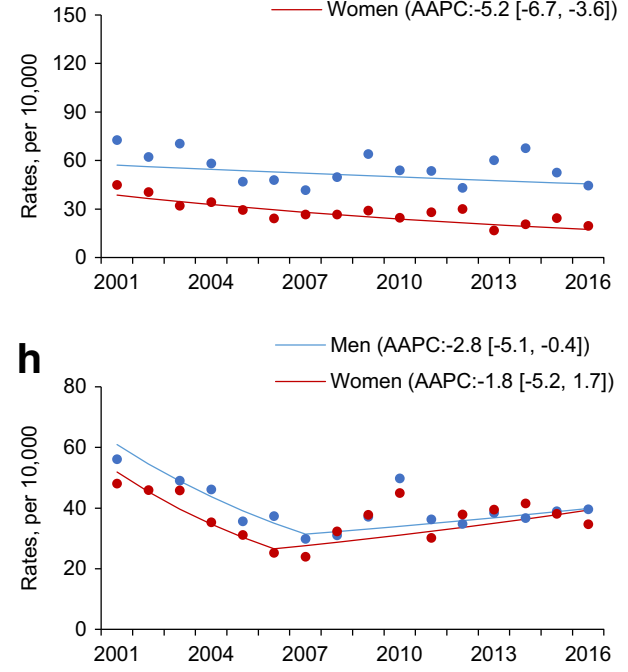

and sepsis but not for influenza, kidney infection or osteomyelitis due to the small numbers of admissions. The rates of hospitalisation for most infection types declined over time in the older age groups (65-74 years and $\geq 75$ years) in both sexes, except for pneumonia for which the rates were unchanged between 2001 and 2016 (ESM Tables 4, 5). Over the surveillance period, the younger age groups (20-44 years and 45-64 years) experienced either an increase or no change in the rates of hospitalisation for most infection types, except tuberculosis for which the rates decreased in both age groups in men and in women aged 45-64 years (ESM Tables 4, 5).

Trends in rates of infection-related hospitalisation in people without diabetes Between 2007 and 2016, 322,589 hospitalisation for pneumonia, 27,679 for influenza, 33,344 for tuberculosis, 15,392 for kidney infections and 50,271 for sepsis were recorded in people without diabetes (ESM Tables 6, 7). During this time, the age-standardised annual 
rates of hospitalisation for influenza increased, rates for tuberculosis decreased and rates for pneumonia were static in both sexes, with no joinpoints identified (ESM Table 8). Rates of hospitalisation for kidney infection and sepsis increased linearly in women and were stable in men (ESM Table 8). Agestratified rates of hospitalisation were calculated for pneumonia, tuberculosis and sepsis, but not for influenza and kidney infection due to small numbers. Rates for pneumonia and sepsis mostly increased from 2007 to 2016 in the age groups of 20-44 years and 45-64 years, were unchanged in those aged 65-74 years, and mostly decreased in the oldest age group (ESM Table 8). Declines in rates for tuberculosis were detected in almost all age groups (ESM Table 8).

\section{Comparison of rates of infection-related hospitalisation} between people with and without diabetes Compared with people without diabetes, those with diabetes had higher rates of hospitalisation for infective causes with age-adjusted rate ratios ranging from $1.3(95 \% \mathrm{CI} 1.3,1.4)$ for pneumonia to 4.9 $(95 \%$ CI 3.9, 6.2) for kidney infection in men, and from 1.4 $(95 \%$ CI $1.3,1.4)$ for pneumonia to $3.2(95 \% 2.8,3.7)$ for kidney infection in women, in 2016 (Table 2 and ESM Tables 9,10). The differences in hospitalisation rates between people with and without diabetes were the greatest in the youngest age group of 20-44 years and became smaller with increasing age (Table 2).

Between 2007 and 2016, a decrease in the rate ratios of hospitalisation for pneumonia was detected for both sexes ( $p<0.001$ in men, $p=0.016$ in women) (Table 2). In men, the rate ratio of hospitalisation for influenza decreased $(p=$ $0.029)$ and that for kidney infection increased $(p=0.031)$ (Table 2). No trends in rate ratios of hospitalisation for tuberculosis and sepsis were observed in either sex (Table 2).

\section{Discussion}

In this population-based analysis of trends in hospitalisation for infection among people with diabetes in Hong Kong, we report four major observations. First, rates of hospitalisation were stagnant for the majority of infection types and have increased for influenza in people with diabetes. Second, there was an age differential in the trends for hospitalisation for infection. In the young to middle-aged groups, hospitalisation rates for most infection types increased or remained unchanged, while in the older age groups, infection rates tended to decline over time. Third, the trends observed among people with diabetes were largely similar to people without diabetes in Hong Kong. Fourth, rates of hospitalisation for all infection types remained consistently higher in people with vs without diabetes throughout the observation period, and the relative differences in rates were the widest in the youngest age group and smallest in the oldest age group.
Unchanged or increasing rates of hospitalisation for common diabetes-related infections People with diabetes are more prone to develop infections because diabetes is associated with other conditions, such as obesity, peripheral sensory neuropathy, chronic kidney disease, peripheral artery disease, stroke and congestive heart failure, that are independent risk factors for infection $[11,12]$. Several studies have also identified blood glucose control as a risk determinant for hospital admission with an infective cause, independent of confounders including age, smoking and comorbidities [4, $11,18]$. In many developed regions, including Hong Kong, the rates of diabetes-related morbidities have fallen dramatically in the past 10-20 years, driven by improvements in blood glucose control and progresses in medical care [13-15]. It is therefore surprising to find static or even increasing rates of hospitalisation for common infection types despite declining prevalence of upstream risk factors. In the present study, rates of hospitalisation for infections of the skin, musculoskeletal system and kidney remained unchanged from 2001 to 2016, with increasing trends for sepsis after 2006/2007. Over a comparable study period between 2000 and 2015, population surveillance conducted in the USA detected increasing rates of hospitalisation for these infection types in people with diabetes [2]. The reason for the divergent trends of kidney infection and urinary tract infection is unclear, but it is possible that the increasing tendency to treat less-severe urinary tract infection in the outpatient setting could contribute to these observations. Discrepancies in coding could also account for the differences. Overall, our results suggest that the achieved improvements in blood glucose and other risk factors might not have been sufficient to prevent infection and progression to adverse outcome. Diabetes education covers various aspects of foot care to prevent foot infection but measures to reduce the risks of other clinically important infections might not have been adequately addressed.

Infection rates are influenced by other factors, such as changing pathogenicity of the infectious agents, development of resistance to antimicrobial drugs, public health measures and clinical practices, that apply to the entire population irrespective of diabetes status. In the present study, people with and without diabetes experienced marked decline in hospitalisation for tuberculosis, reflecting the successes of public health measures and medical treatment, although the relative difference in rates of tuberculosis between people with and without diabetes has not changed over time. Similarly, the rate ratios of hospitalisation due to kidney infection or sepsis by diabetes status have remained constant or even increased. In the study in the USA, a rise in the rate ratios for these infection types as well as infections of the skin and musculoskeletal system were also found [2]. Sustained risk differential indicates that the increased risks of serious infection in people with diabetes were unaltered by advances in diabetes 


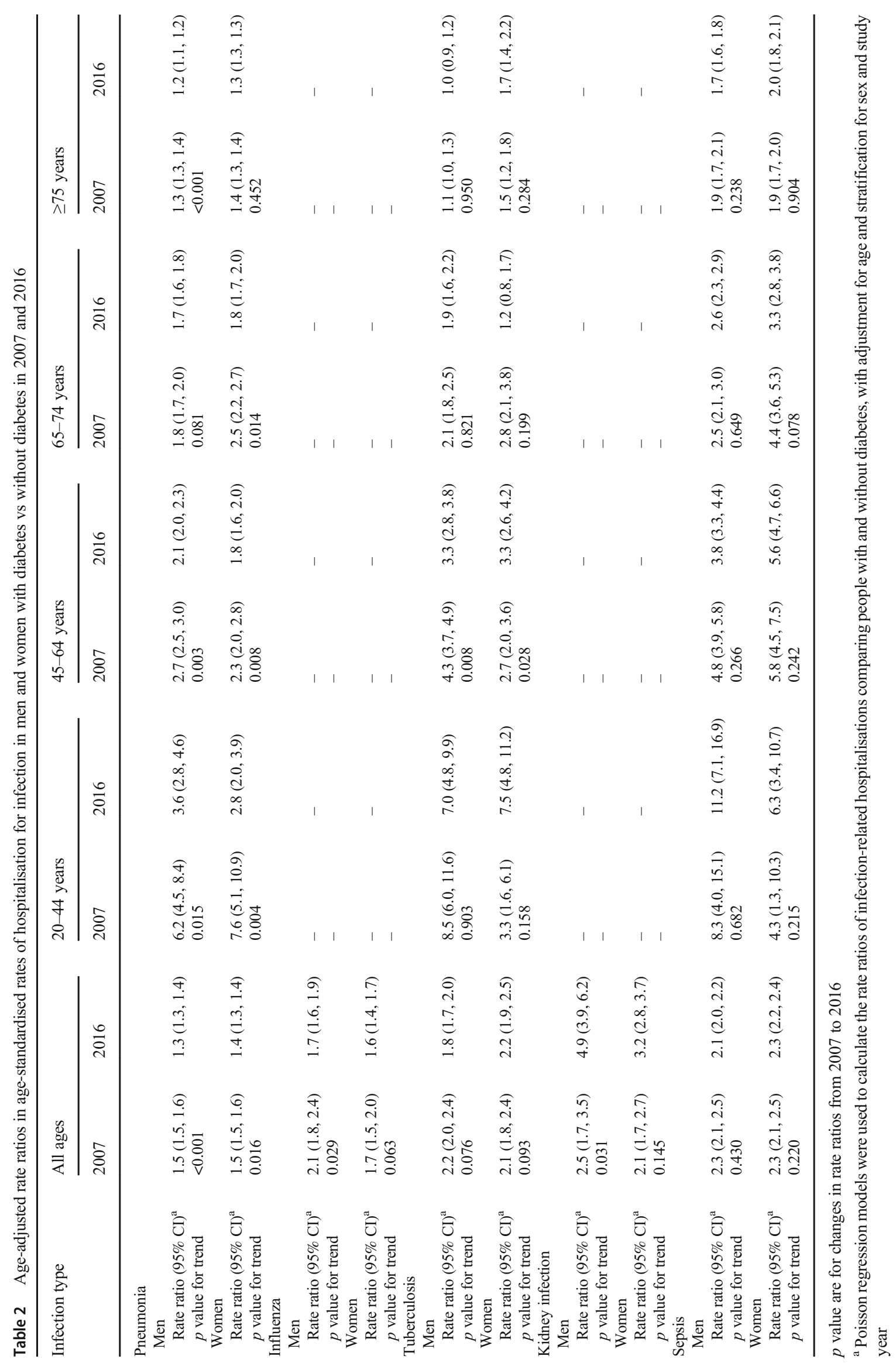


therapeutics and that much room exists to narrow the gap in infection rates between people with and without diabetes.

Trends in rates of hospitalisation for pneumonia and influenza The trends in hospitalisation for pneumonia and influenza deserve further consideration. We detected an increase in rates of hospitalisation for pneumonia from 2001 to 2004/2005, after which the rates decreased in men and were unchanged in women. In early- to mid-2003, Hong Kong experienced the SARS epidemic, in which 1750 local residents were infected with the coronavirus and 286 people died [19]. An increase in health awareness among the general population and a lower medical threshold for hospital admission during the immediate post-SARS period most likely explain the spike in rates of hospitalisation for pneumonia in 2004/2005. Stabilisation in rates thereafter could be partly attributed to measures taken by the local health authority to improve cleanliness of housing estates and districts, as well as widespread campaigns to promote personal hygiene [19]. Stabilisation in rates for pneumonia was also noted in people without diabetes and, contrary to other common infection types, the relative difference in rates between people with and without diabetes diminished during the study period. The reasons are unclear but more liberal use of renin-angiotensin-aldosterone system inhibitors and statins, which have been shown to lower the risks of pneumonia, among people with diabetes might contribute to these observations [20,21].

In contrast to the stable trends for pneumonia, we observed rising trends in hospitalisation for influenza in people with and without diabetes. Diabetes is associated with a greater hazard of severe influenza $[22,23]$. International guidelines recommend annual vaccination against influenza in people with diabetes [24], although whether people with diabetes derive the same level of protection as do people without diabetes remains inconclusive [25]. The Hong Kong Government influenza vaccination programme launched in 2004 prioritised free or subsidised immunisation to all people with diabetes [26]. Despite widespread promotion of the programme, only $12 \%$ of the general population were vaccinated in the most recent survey in 2015/16, although we have no information on the vaccination rates in people with diabetes [27]. The dramatic increase in annual rates of hospitalisation for influenza in both people with and without diabetes in Hong Kong could reflect increases in transmissibility and pathogenicity of the virus, compounded by increasing population density and overcrowding. Changes in clinical practice, in particular more frequent testing for influenza in people presenting with respiratory tract symptoms, could also explain the escalating trend.

\section{Age differential in trends of hospitalisation for infection In} the present study, we found that rates of hospitalisation for most infections decreased in the older age groups (aged $\geq 65$ years) but were stable or increased among the young and middle-aged groups. Our results are consistent with the study in the USA, which reported rising trends for hospitalisation rates for many infection types in the younger age groups (aged 18-44 years) with diabetes despite the decline in their older counterparts [2]. The lack of improvement shown in trends in the young people is evident across a range of diabetes-related complications and mortality rates in earlier analyses $[17,28]$. Young people with diabetes have higher blood glucose levels than older people due to a combination of poorer treatment adherence and therapeutic inertia [29]. Young people also tend to delay seeking medical care, leading to more severe and clinically advanced presentation of medical conditions including infection. It is conceivable that these health-compromising behaviours of young people has not changed significantly over time, and that current approaches to managing diabetes have not been effective in improving health indices in this group. The differential infection risk associated with diabetes in younger vs older age groups is likely due to the lower background rates among the young general population. Nonetheless, the three- to fourfold increase in rates of hospitalisation for pneumonia, sevenfold increase in rates for tuberculosis, and six- to 11-fold increase in rates for generalised sepsis in young people with diabetes aged 20-44 years relative to their age-matched counterparts without diabetes highlights the unmet needs in these young individuals and calls for focused preventive strategies.

Limitations This study has several limitations. First, the HKDSD comprises people who attended public hospitals and clinics operated by the HKHA and does not include people who presented to the private health sector. The HKHA covers about $95 \%$ of health services in Hong Kong and the exclusion of the small proportion of people with diabetes treated in private should have minimal impact on trend estimates. Second, the HKDSD is an administrative database subjected to issues of validity. For instance, people who were prescribed glucoselowering drugs, such as metformin, for conditions other than diabetes could be included although the number is deemed small. Furthermore, we could not differentiate type 1 diabetes from type 2 diabetes in the HKDSD, although over $98 \%$ of Hong Kong Chinese with diabetes have type 2 diabetes [30]. Third, the increasing number of people with newly diagnosed diabetes each year could affect the trend estimates. In the sensitivity analysis, excluding those newly included in the HKDSD each study year did not yield any significant difference in the trends of infection-related hospitalisation. Fourth, we relied on diagnostic codes listed as principal diagnosis for event count and infections recorded as a secondary diagnosis were not included, leading to potential under-estimation of event rates. This limited the scope of the study to 
evaluate the trends in rates of nosocomial infections, which are more likely to be entered as secondary diagnosis. Fifth, people with diabetes could be more readily admitted to hospital than people without diabetes due to the greater care-seeking tendency of the former group and lower threshold for admitting people with chronic diseases such as diabetes. Trend estimates could be affected if the hospitalisation threshold has changed significantly over time. This was evident for hospitalisation for pneumonia around the time of the SARS epidemic. Sixth, data for hospital admission of people without diabetes were not available before 2007 and the available data only captured hospitalisation for a limited range of infection diagnoses. The comparison of rates between people with and without diabetes across the full surveillance period was not possible, resulting in a significantly shorter time period for trend analysis. Seventh, analysis of people without diabetes was based on aggregate data. In addition, different ICD coding systems were used to identify infectionrelated hospitalisation in people with diabetes and in people without diabetes. This would have affected the estimates of incidence rate ratios, although our estimates are similar to figures reported by others. Last, the study described the trends in Hong Kong and results may not be generalisable to other regions.

Conclusions We observed unchanged or increasing rates of hospitalisation for most types of diabetes-related infection, except for decline in rates for tuberculosis and urinary tract infection, among people with diabetes in Hong Kong over a 16-year period. Improvements in diabetes care have not translated to narrowing of relative differences in rates of hospitalisation for most infection types when comparing people with and without diabetes during this time. The recent COVID-19 global outbreak has revealed the toll of diabetes and other chronic diseases on the healthcare system during acute emergencies. Greater efforts directed towards infection prevention and control in people with diabetes are urgently needed to reduce their risks of developing and progressing to serious infections.

Acknowledgements We acknowledge the HKHA for providing anonymised data for research. In addition, we acknowledge the Department of Health of Hong Kong Special Administrative Region for providing summary data of hospitalisation of the general population in Hong Kong. This work has received no external funding.

Data availability Data supporting the results of this study are hosted at the HKHA and at the Department of Health of Hong Kong Special Administrative Region.

Funding This research received no specific grant from any funding agency in the public, commercial or not-for-profit sectors.

Authors' relationships and activities The authors declare that there are no relationships or activities that might bias, or be perceived to bias, their work.
Contribution statement AOYL contributed to conception and design, acquisition and interpretation of data, and drafted the article. HW contributed to conception and design, and acquisition and analysis of data. ESHL, AY, WYS, EC, APSK, DSCH, RCWM and JCNC contributed to conception and design, and acquisition and interpretation of data. All authors revised the article critically and approved the final version. AOYL is the guarantor of this work, has full access to all the data in the study and takes responsibility for the integrity of the data and the accuracy of the data analysis.

\section{References}

1. Kornum JB, Thomsen RW, Riis A, Lervang HH, Schønheyder HC, Sørensen HT (2008) Diabetes, glycemic control, and risk of hospitalisation with pneumonia: a population-based case-control study. Diabetes Care 31(8):1541-1545. https://doi.org/10.2337/ dc08-0138

2. Harding JL, Benoit SR, Gregg EW, Pavkov ME, Perreault L (2020) Trends in rates of infections requiring hospitalisation among adults with versus without diabetes in the U.S., 2000-2015. Diabetes Care 43(1):106-116. https://doi.org/10.2337/dc19-0653

3. Carey IM, Critchley JA, DeWilde S, Harris T, Hosking FJ, Cook DG (2018) Risk of infection in type 1 and type 2 diabetes compared with the general population: a matched cohort study. Diabetes Care 41(3):513-521. https://doi.org/10.2337/dc17-2131

4. Benfield T, Jensen JS, Nordestgaard BG (2007) Influence of diabetes and hyperglycaemia on infectious disease hospitalisation and outcome. Diabetologia 50(3):549-554. https://doi.org/10.1007/ s00125-006-0570-3

5. Shah BR, Hux JE (2003) Quantifying the risk of infectious diseases for people with diabetes. Diabetes Care 26(2):510-513. https://doi. org/10.2337/diacare.26.2.510

6. Magliano DJ, Harding JL, Cohen K, Huxley RR, Davis WA, Shaw JE (2015) Excess risk of dying from infectious causes in those with type 1 and type 2 diabetes. Diabetes Care 38(7):1274-1280. https:// doi.org/10.2337/dc14-2820

7. Yang JK, Feng Y, Yuan MY et al (2006) Plasma glucose levels and diabetes are independent predictors for mortality and morbidity in patients with SARS. Diabet Med 23(6):623-628. https://doi.org/10. 1111/j.1464-5491.2006.01861.x

8. Yang X, Yu Y, Xu J et al (2020) Clinical course and outcomes of critically ill patients with SARS-CoV-2 pneumonia in Wuhan, China: a single-centered, retrospective, observational study. Lancet Respir Med 8(5):475-481. https://doi.org/10.1016/S22132600(20)30079-5

9. Stegenga ME, van der Crabben SN, Blümer RM et al (2008) Hyperglycaemia enhances coagulation and reduces neutrophil degranulation, whereas hyperinsulinemia inhibits fibrinolysis during human endotoxemia. Blood 112(1):82-89. https://doi.org/ 10.1182/blood-2007-11-121723

10. Delamaire M, Maugendre D, Moreno M, Le Goff MC, Allannic H, Genettet B (1997) Impaired leucocyte functions in diabetic patients. Diabet Med 14(1):29-34. https://doi.org/10.1002/(SICI)10969136(199701)14:1<29::AID-DIA300>3.0.CO;2-V

11. Luk AO, Lau ES, Cheung KK et al (2017) Glycaemia control and the risk of hospitalisation for infection in patients with type 2 diabetes: Hong Kong Diabetes Registry. Diabetes Metab Res Rev 33(8): e2923. https://doi.org/10.1002/dmrr.2923

12. Cardoso CR, Salles GF (2007) Macro and microvascular complications are determinants of increased infection-related mortality in 
Brazilian type 2 diabetes mellitus patients. Diabetes Res Clin Pract 75(1):51-58. https://doi.org/10.1016/j.diabres.2006.04.008

13. Luk AO, Hui EM, Sin MC et al (2017) Declining trends of cardiovascular-renal complications and mortality in type 2 diabetes: the Hong Kong Diabetes Database. Diabetes Care 40(7):928-935. https://doi.org/10.2337/dc16-2354

14. Gregg EW, Li Y, Wang J et al (2014) Changes in diabetes-related complications in the United States, 1990-2010. N Engl J Med 370(16):1514-1523 https://www.nejm.org/doi/10.1056/ NEJMoa1310799

15. Vamos EP, Millett C, Parsons C, Aylin P, Majeed A, Bottle A (2012) Nationwide study on trends in hospital admissions for major cardiovascular events and procedures among people with and without diabetes in England, 2004-2009. Diabetes Care 35(2):265-272. https://doi.org/10.2337/dc11-1682

16. Hospital Authority (2016) HA annual report 2015-2016. Available from https://www.ha.org.hk/visitor/ha_visitor_index.asp?Content ID $=235572 \&$ Lang=ENG. Accessed 21 April 2020

17. Wu H, Lau ESH, Yang A et al (2020) Trends in diabetes-related complications in Hong Kong, 2001-2016: A retrospective cohort study. Cardiovasc Diabetol 19(1):60. https://doi.org/10.1186/ s12933-020-01039-y

18. Critchley JA, Carey IM, Harris T, DeWilde S, Hosking FJ, Cook DG (2018) Glycemic control and risk of infections among people with type 1 or type 2 diabetes in a large primary care cohort study. Diabetes Care 41:2127-2135. https://doi.org/10.2337/dc18-0287

19. Abdullah AS, Tomlinson B, Cockram CS, Thomas GN (2003) Lessons from the severe acute respiratory syndrome outbreak in Hong Kong. Emerg Infect Dis 9(9):1042-1045. https://doi.org/10. 3201/eid0909.030366

20. Caldeira D, Alarcăo J, Vaz-Carneiro A, Costa J (2012) Risk of pneumonia associated with use of angiotensin converting enzyme inhibitors and angiotensin receptor blockers: systematic review and meta-analysis. BMJ 345:e4260. https://doi.org/10.1136/bmj.e4260

21. Mortensen EM, Nakashima B, Cornell J et al (2012) Populationbased study of statins, angiotensin II receptor blockers, and angiotensin-converting enzyme inhibitors on pneumonia-related outcomes. Clin Infect Dis 55(11):1466-1473. https://doi.org/10. 1093/cid/cis733

22. Lau D, Eurich DT, Majumdar SR, Katz A, Johnson JA (2014) Working-age adults with diabetes experience greater susceptibility to seasonal influenza: a population-based cohort study. Diabetologia 57(4):690-698. https://doi.org/10.1007/s00125-0133158-8

23. Allard R, Leclerc P, Tremblay C, Tannenbaum TN (2010) Diabetes and the severity of pandemic influenza A (H1N1) infection. Diabetes Care 33(7):1491-1493. https://doi.org/10.2337/dc092215

24. American Diabetes Association (2020) Comprehensive medical evaluation and assessment of comorbidities: Standards of Medical Care in Diabetes -2020. Diabetes Care 43(Suppl 1):S37-S47. https://doi.org/10.2337/dc20-S004

25. Remschmidt C, Wichmann O, Harder T (2015) Vaccines for the prevention of seasonal influenza in patients with diabetes: systematic review and meta-analysis. BMC Med 13:53. https://doi.org/10. 1186/s12916-015-0295-6

26. Centre for Health Protection Scientific Committee on Vaccine Preventable Diseases (2015) Recommendations on seasonal influenza vaccination for the 2015/16 season. Available from https:// www.chp.gov.hk/files/pdf/short_version_of recommendations on_seasonal_influenza_vaccination_for_the_2015_16_final.pdf. Accessed 21 April 2020

27. Chan YW, Wong ML, Au KW, Chuang SK (2019) Seasonal influenza vaccine effectiveness at primary care level, Hong Kong SAR, 2017/18 winter. Hum Vaccin Immunother 15(1):97-101. https:// doi.org/10.1080/21645515.2018.1514222

28. Gregg EW, Hora I, Benoit SR (2019) Resurgence in diabetesrelated complications. JAMA 321(19):1867-1868. https://doi.org/ 10.1001/jama.2019.3471

29. Yeung RO, Zhang Y, Luk A et al (2014) Metabolic profiles and treatment gaps in young-onset type 2 diabetes in Asia (the JADE programme): a cross-sectional study of a prospective cohort. Lancet Diabetes Endocrinol 2(12):935-943. https://doi.org/10.1016/ S2213-8587(14)70137-8

30. Luk AOY, Ke C, Lau ESH et al (2020) Secular trends in incidence of type 1 and type 2 diabetes in Hong Kong: a retrospective cohort study. PLoS Med 17(2):e1003052. https://doi.org/10.1371/journal. pmed. 1003052

Publisher's note Springer Nature remains neutral with regard to jurisdictional claims in published maps and institutional affiliations. 